\title{
Lytic Bacteroides uniformis bacteriophages exhibiting host tropism congruent with diversity generating retroelement
}

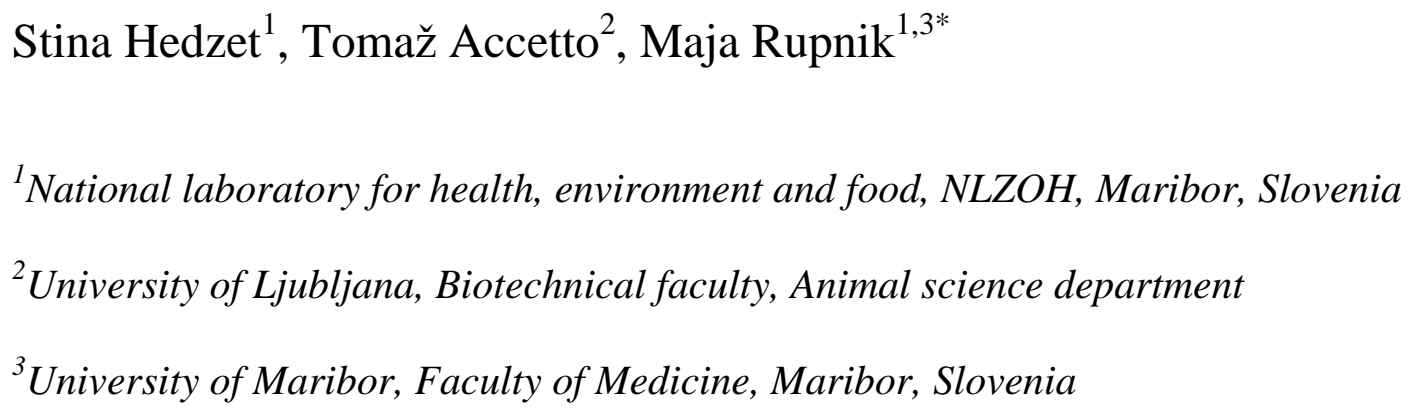




\section{Abstract}

32 Intestinal phages are abundant and important component of gut microbiota, but our knowledge

33 remains limited to only a few isolated and characterized representatives targeting numerically

34 dominant gut bacteria. Here we describe isolation of human intestinal phages infecting

35 Bacteroides uniformis. Bacteroides is one of the most common bacterial groups in the global

36 human gut microbiota, however, to date not many Bacteroides specific phages are known. Phages

37 isolated in this study belong to a novel viral genus, Bacuni, within Siphoviridae family and

38 represent the first lytic phages, genomes of which encode diversity generating retroelements

39 (DGR). This region is assumed to promote phage adaptation to the rapidly changing

40 environmental conditions and to broaden its host range. Three isolated phages showed $99,83 \%$

41 genome identity but infected distinct $B$. uniformis strains. The tropism of Bacuni phages appeared

42 to be dependent on the interplay of DGR mediated sequence variations of phage fimbrial tip

43 proteins and mutations in host genes coding for outer-membrane proteins. We found prophages

44 with up to $85 \%$ aa similarity to Bacuni phages in the genomes of $B$. acidifaciens and Prevotella

45 sp.. Despite the abundance of Bacteroides within human microbiome, we found Bacuni phages

46 only in a limited subset of published gut metagenomes.

\section{Importance}

49 The lack of common marker gene in viruses require a precise characterization of diverse isolated

50 phages to enhance metagenomic analyses and to understand their role in gut microbiota. Here we

51 report the isolation of phages representing a new genus with characteristics so far not known or

52 rarely described in intestinal phages. They are the first lytic phages specific for Bacteroides

53 uniformis, a bacterial representative of the prevalent genus in the gut of humans and animals.

54 Additionally, they are the first lytic phages containing specific regions (diversity generating 
55 retroelement) that putatively influence host tropism. The ability to switch constantly the targeted

56 populations of the host species could provide an evolutionary advantage to these bacteriophages

57 and may affect intra species diversity.

58

59

60

61 Keywords: gut, Bacteroides, virome, lytic phage, prophage, Diversity Generating Retroelement

62

63 


\section{Introduction}

Intestinal viruses and their impact on human health are a neglected component of the widely studied gut microbiota. Bacteriophages (phages) exhibit different life styles and play an important

67 role in shaping bacterial diversity and composition of the intestinal microbiota through predation

68 and horizontal gene transfer ${ }^{1,2}$. Sequencing-based metagenomic studies have enabled insight into

69 this complex viral reservoir revealing genetically very diverse phages ${ }^{1,3-5}$.

70 Virome metagenomic studies encounter several difficulties. The vast majority (75-99\%) of

71 sequencing reads does not correspond to any matches in the existing viral databases ${ }^{3}$. Viruses

72 lack universal marker genes, while standardized protocols for sample preparations and analysis

73 are not yet established ${ }^{1}$. To decipher gut virome and to connect biological characteristics with

74 metagenomic data, cultivation of intestinal phages and their associated hosts remains crucial. A

75 great number of intestinal phages infect anaerobic bacteria, which are challenging to cultivate;

76 isolated and characterized phages are therefore sparse.

77 Despite these difficulties, several phages and prophages were lately described in different 78 anaerobic gut microbiota representatives. In silico discovered viral clade, CrAss-like phages, is

79 presumably present in $50 \%$ of Western individuals and can represent up to $90 \%$ of viral

80 metagenomics reads per individual sample ${ }^{6,7}$. Prediction of suspected Bacteroides sp. host was

81 confirmed by isolation of a CrAss-like phage, Crass001, that infects Bacteroides intestinalis and

82 exhibits a podovirus-like morphology ${ }^{8}$. Its life style has yet to be elucidated. CrAss-like phages

83 are a group of genetically highly diverse phages making additional Bacteroides sp. or other

84 bacteria probable hosts ${ }^{9}$. Lysogenic (temperate) phages have been identified in genomes of 85 Fecalibacterium prausnitzii ${ }^{10}$ and Bacteroides dorei ${ }^{11}$. Extremely large gut phage genomes

$86(540 \mathrm{~kb})$, named Lak phages, that presumably infect Prevotella sp. were also recovered from gut 
87 metagenomes ${ }^{12}$. Recently, a study of temperate phage-bacteria interactions in mice gut showed

88 that Roseburia intestinalis prophages influence temporal variations in composition of gut 89 microbiota ${ }^{13}$. Additionally to Bacteroides dorei Hankyphage ${ }^{11}$ and CrAss001 ${ }^{8}$, four phages

90 infecting different species within Bacteroides genus were isolated and sequenced. Phages B40-8

$91{ }^{14}$ and B124-14 ${ }^{15}$ infect Bacteroides fragilis, while phages $\phi \mathrm{Brb} 01$ and $\phi \mathrm{Brb} 02$, originating from

92 sewage, infect Bacteroides sp. bacterial hosts isolated from rumen fluid ${ }^{16}$. However, compared to

93 more than 150 phages infecting $E$. coli isolated from various biomes and clinical settings ${ }^{17}$, there

94 are few reported bacteriophages infecting species from the genus Bacteroides, which account for

95 roughly $30 \%$ of all bacteria in an average human intestine ${ }^{18}$.

96 Diversity-generating retroelements (DGRs) are genetic elements, composed of template-

97 dependent reverse transcriptase and accessory proteins that produce mutations in targeted genes

98 with variable repeats. This introduces the variability in the target proteins ${ }^{19}$. DGR mechanism

99 was first described in Bordetella phage BPP-1, in which mutations target phage tail fiber gene via 100 "mutagenic retrohoming" to enable bacterial host species switching ${ }^{20,21}$. Phage-encoded DGRs 101 were also found in genomes of isolated temperate phages of intestinal B. dorei ${ }^{11}$ and $F$. 102 prausnitzii ${ }^{10}$. Moreover, DGRs were detected in defined prophage regions of bacteria belonging 103 to Bacteroidetes, Proteobacteria and Firmicutes, obtained from human-gut associated 104 metagenomes and bacterial genomes ${ }^{11}$.

105 Understanding the intestinal virome depends on the number of isolated, sequenced, and 106 characterized bacteriophages and their associated hosts. The aim of the present study was to 107 obtain and characterize the phages targeting abundant gut bacteria from Bacteroides genus, and 108 to contribute to the insight of the "viral dark matter" 22 of the interactions of bacteria and viruses 
in human gut. Additionally, the study provides bioinformatic evidence that the host range of

110 isolated phages may very well be mediated by a DGR.

\section{Materials and methods}

113 Isolation of bacterial strains from human fecal sample

114 Fecal sample, obtained from a healthy volunteer was aliquoted and further processed or stored at

$115-80^{\circ} \mathrm{C}$. The complete isolation of bacterial strains and preparation of fecal suspension was carried 116 out in an anaerobic workstation at $37^{\circ} \mathrm{C}$ (Don Whitley Scientific).

117 Dilutions of homogenized fecal suspension (20\%), made from fresh feces and pre-reduced 118 anaerobic YBHI culture media (Brain-heart infusion media, supplemented with $0.5 \%$ yeast 119 extract (BioLife) and 20\% of rumen fluid) were plated on YBHI agar. After 72 hours of anaerobic 120 incubation at $37^{\circ} \mathrm{C}$ single colonies were randomly chosen and isolated on YBHI plates to obtain

121 pure bacterial cultures. Isolates were identified by mass spectrometry (MALDI-TOF Biotyper 122 System, Bruker Daltonik, Bremen, Germany). Identification of Bacteroides strains was confirmed 123 by $16 \mathrm{~S}$ rRNA gene sequencing amplified with primers $27 \mathrm{feb}$ to $1495 \mathrm{revb}{ }^{23}$ and analyzed with 124 RDP Classifier ${ }^{24}$.

125 Isolated Bacteroides strains (Data set S1) were then used in phage screening experiment and host 126 range experiment.

129 Fecal sample used for the phage isolation was not identical as used for bacterial strain isolation 130 but was retrieved from the same healthy volunteer. Fresh fecal material $(5 \mathrm{~g})$ was resuspended in $13150 \mathrm{~mL}$ of SM buffer with vigorous vortexing for 20 minutes. SM buffer contained $100 \mathrm{mM} \mathrm{NaCl}$, 
133 down on ice, fecal suspension was centrifuged twice at $5400 \times \mathrm{g}\left(4^{\circ} \mathrm{C}\right)$. Supernatant was filtered

134 twice through $0.2 \mu \mathrm{m}$ pore cellulose acetate (CA) syringe membrane filters (Filtropur, Starsted).

135 Sterile filtrate of homogenized fecal sample (fecal water) was stored at $+4^{\circ} \mathrm{C}$ until further use.

136 Phages were initially enriched in Bacteroides cultures. Ten different Bacteroides strains in

137 stationary phase $(1 \mathrm{~mL})$ were subcultured into $9 \mathrm{~mL}$ of liquid sABB (Anaerobe Basal Broth,

138 Thermo Fisher Scientific, supplemented with $\mathrm{MgSO}_{4}(0.12 \mathrm{mM})$ and $\left.\mathrm{CaCl}_{2}(1 \mathrm{mM})\right)$. For phage

139 enrichment, $1 \mathrm{~mL}$ of fecal water was added to the inoculated media and incubated for 24 hours at

$14037^{\circ} \mathrm{C}$. Subsequently, $3 \mathrm{~mL}$ of culture media were removed and centrifuged at $5400 \times \mathrm{g}\left(4^{\circ} \mathrm{C}\right)$.

141 Supernatant was syringe-filtered $(0.2 \mu \mathrm{m}$ pore, Starsted $)$ and added to $9 \mathrm{~mL}$ of fresh sABB

142 media, inoculated with Bacteroides strain in stationary phase like described before. The

143 procedure was again repeated after 24 hours. The final sterile supernatant was refrigerated $\left(4^{\circ} \mathrm{C}\right)$

144 until further used in double-agar-layer method. Maximal storage duration was 72 hours.

\section{Phage isolation from enrichment co-cultures with Bacteroides}

146 Spot assay on a double-agar-layer (DAL) was used for phage isolation from enrichment cultures.

147 Bacteroides strains, cultivated in liquid sABB, were sampled at two different time points with 148 optical densities $\mathrm{OD}_{620} 0,2$ (T1) and $\mathrm{OD}_{620} 0,5$ (T2) for further use in DAL assay. For each time 149 point 10 -fold dilutions were made and $200 \mu \mathrm{L}$ of each dilution was mixed with $3 \mathrm{ml}$ soft agar that was kept anaerobically at $47^{\circ} \mathrm{C}(\mathrm{sABB})$ and poured on the prereduced sABB agar basal plates.

151 After solidification 10-fold dilutions of supernatant filtrates of phage enrichment cultures $(10 \mu \mathrm{L})$

152 were spotted on solidified agar. After $24 \mathrm{~h}$ of incubation plates were checked for potential lysis

153 zones. The top agar with clear zones was harvested with an inoculation loop and stored in $100 \mu \mathrm{L}$ 
154 of SM puffer for $18-24 \mathrm{~h}$ at $4^{\circ} \mathrm{C}$, followed by centrifugation $(13000 \times \mathrm{g}, 5 \mathrm{~min})$. Supernatant was

155 then used for further steps in phage purification and characterization.

156 Phages were purified from the stored spot assay supernatants by three consecutive single plaque

157 isolation cycles using the corresponding bacterial host strain. Bacteroides culture $(200 \mu \mathrm{L})$ in $\log$

158 growth phase was mixed with 10 -fold dilutions of lysis zone supernatant and $2.5 \mathrm{~mL}$ of $\mathrm{sABB}$

159 soft agar and poured onto sABB agar basal plates, allowed to solidify, and incubated at $37^{\circ} \mathrm{C}$.

160 After 18-24h incubation, a single plaque was picked with pipette tip, transferred to SM buffer

$161(100 \mu \mathrm{L})$ and left overnight at $4^{\circ} \mathrm{C}$. After $18-24 \mathrm{~h}$, phage lysates were centrifuged $(13000 \times \mathrm{g}, 5$

$162 \mathrm{~min}$ ) and used in a plaque assay.

163 Preparation of phage stock suspensions, EM characterization and host range

164 Each isolated phage in SM buffer $(100 \mu \mathrm{L})$ and $200 \mu \mathrm{L}$ of respective host bacterial culture $\left(10^{7}\right.$

$165 \mathrm{cfu} / \mathrm{ml}$ ) was mixed into $3 \mathrm{~mL}$ soft agar, poured on solid agar plate, and incubated up to $24 \mathrm{~h}$ at

$16637^{\circ} \mathrm{C}$. Subsequently, SM buffer $(4 \mathrm{~mL})$ was gently poured on confluently lysed top agar. Plates

167 were further incubated at $37^{\circ} \mathrm{C}$ for 4 hours with gentle shaking. Top agar and the remains of SM

168 buffer were scraped and centrifuged at $5400 \times \mathrm{g}\left(4^{\circ} \mathrm{C}\right)$. The supernatant was filtered through 0.2

$169 \mu \mathrm{m}$ pore CA syringe membrane filters (Filtropur, Starsted). Prepared phage suspension was

170 transferred to U-formed centrifuge tubes suitable for ultra-centrifugation $(25000 \times \mathrm{g}, 120 \mathrm{~min}$,

$\left.1714{ }^{\circ} \mathrm{C}\right)\left(\right.$ Beckman Coulter, Optima $\left.{ }^{\mathrm{TM}} \mathrm{MAX}-\mathrm{XP}\right)$. Pellets were resuspended in $200 \mu \mathrm{L}$ of SM buffer

172 and phage stock suspensions were stored at $4^{\circ} \mathrm{C}$ and $-80^{\circ} \mathrm{C}$.

173 Transmission electron microscopy was performed at National institute for biology, Ljubljana, 174 Slovenia). 
175 Host range of isolated phages was tested with the double-agar-layer assays using 12 Bacteroides

176 strains belonging to four species (Data set S1).

177 Lysogen formation assay

178 Each isolated phage was cultivated with its respective host strain. Plates with formed plaques in

179 plaque assay were incubated in anaerobic chamber at $37^{\circ} \mathrm{C}$ for additional 72 hours, to allow the

180 growth of potential lysogenic strains. From the plaques formed on double-layer agar, bacterial

181 cultures were isolated with a sterile needle or small pipette tip and inoculated on sABB agar

182 plates to obtain pure cultures. At least 12 strains were isolated per tested bacteriophage.

183 Sensitivity of obtained strains for isolated phages was tested with DAL spot assay described

184 above (Figure S4).

Phage and bacterial genome sequencing

186 Phage lysate $(200 \mu \mathrm{L})$ with app. $10^{9} \mathrm{pfu} / \mathrm{ml}$ was treated with DNase I (Sigma Aldrich) at the final 187 concentration of $0.02 \mathrm{mg} / \mathrm{ml}$ and $0.05 \mathrm{mg} / \mathrm{mL}$ RNAse A (Sigma Aldrich) and incubated for $2 \mathrm{~h}$ at $18837^{\circ} \mathrm{C}$, followed by $10 \mathrm{~min}$ heat inactivation at $90^{\circ} \mathrm{C}$. Potential presence of host genomic residues

189 was assayed with PCR using primers targeting $16 \mathrm{~S}$ rRNA gene ${ }^{23}$. Phage DNA was extracted 190 with RTP $^{\circledR}$ DNA/RNA Virus Mini Kit following manufacturer's instructions (INVITEK 191 Molecular).

192 Bacteroides DNA was extracted (QIAamp DNA Mini Kit, Qiagen).

193 For phage and bacterial genomes paired-end libraries were generated using the Nextera XT 194 Library preparation kit (IIlumina) and sequenced on MiSeq (Ilumina) with 600-cycle MiSeq 195 ReagentKit v3. 
The quality of the raw sequencing reads was examined by FastQC tool Version 0.11.9 (Babraham

Bioinformatics) ${ }^{25}$. Quality trimming was done by Trimmomatic Version 0.39

198 (USADELLAB.org) ${ }^{26}$ and overlapping paired-end reads were merged by using FLASH

199 software, version 1.2.11 (CBB) ${ }^{27}$. Assembly was performed by SPAdes Assembler, version

$2003.14 .0^{28}$ and the assemblies were examined using Quast version $4.0^{29}$. Genomes were then

201 annotated with Prokka $1.14 .5^{30}$.

\section{Bacteriophage genome annotation}

203 Protein sequences of open reading frames (ORFs), determined with Prokka $1.14 .5^{30}$, were 204 blasted (blastp, NCBI, 2019) against non-redundant protein sequences (nr) database. Conserved 205 protein domains of ORF were predicted with Conserved Domain Search (CDD, NCBI) and Pfam 206 31. Additionally, remote homologues were also detected using PHYRE2 - Protein

207 Homology/analogY Recognition Engine V 2.0 ${ }^{32}$. Presence of signal peptides was analyzed with 208 SignalP-5.0 Server ${ }^{33}$. Remote homologs of phage head-neck-tail module proteins were 209 additionally analyzed on VIRFAM sever ${ }^{34}$. Predicted DGR regions were analyzed with myDGR, 210 a server for identification and characterization of diversity-generating retroelements ${ }^{35}$.

\section{Phage classification and phylogenetic analysis}

212 Phage life style and classification was computationally analyzed using PHACTS program 213 (http://www.phantome.org/PHACTS/index.htm) ${ }^{36}$.

214 vConTACT2 ${ }^{37}$ was used for taxonomic classification using the ViralRefSeq-prokaryotes-v94 215 database. To determine phage DNA packaging and replication strategy, a phylogenetic analysis 216 of amino acid sequences of TerL - terminase large subunit was made. Sequences of TerL were 217 downloaded from NCBI and Pfam databases and aligned using the ClustalW ${ }^{38}$ program. 
218 Phylogenetic tree was then generated with the SeaView Version 5.0.2 ${ }^{39}$ integrated phyML using 219 the maximum likelihood approach and GTR nucleotide substitution model. The resulting 220 dendrogram was then visualized with FigTree v1.4.4 (http://tree.bio.ed.ac.uk/software/figtree/).

222 Identification of shared homologous proteins and prophage regions

223 Based on closest BLASTp hits of determined ORFs, closest relatives were manually predicted 224 and their bacterial host genomes were examined for prophage presence. Ranges of prophage 225 regions were determined based on the $\mathrm{G}+\mathrm{C}$ content, predicted functional annotations of 226 neighboring genes, presence of integrase and other phage specific genes or identification of 227 repeats sites (attL and attR). Sequences of predicted prophage regions were extracted from host 228 genomes using Artemis software version $1.8^{40}$, annotated with Prokka $1.14 .5^{30}$ and applied in 229 comparison using Easyfig ${ }^{41}$. Protein sequences of ORFs of identified prophages were analyzed 230 for conserved protein domains like described above. Gene synteny in different phage functional 231 gene groups was analyzed.

\section{SNP analysis of potential phage target genes}

233 Reads of original phage host (B. uniformis MB18-80) and two derivative strains isolated in 234 lysogeny experiment (MB18-80-K and MB18-80-PH) were mapped to original MB18-80 235 assembly using BBTools ${ }^{42}$. Sorted BAM files were used for calling SNPs sites using the

236 SAMtools verison $0.1 .19^{43}$. Mapped reads and SNP sites were also analyzed relative to MB18-80 237 genome using Artemis software version $1.8^{40}$.

\section{Tandem repeats analysis with direct sequencing}


239 Tandem repeats were located and analyzed with Tandem Repeats Finder ${ }^{44}$. Primers (primer F2, 240 5'-CCTCGGTAATGCTTTCTACG-3'; primer R2, 5'-AGGTAGCCGTAAATGTATCG-3') were

241 constructed using SnapGene software (GSL Biotech LLC, 2004) and were used in a direct Sanger 242 sequencing reaction (40 cycles; using a gDNA as a template and BigDye Terminator v3.1 Cycle 243 Sequencing Kit) to examine if the repeats represent phage genome termini of linear dsDNA 244 phage. Sequencing was performed on 3500 Series Genetic Analyzer (ThermoFisher Scientific) 245 and analyzed with Artemis software version $1.8^{40}$

246 Metagenomic analysis

247 Paired-end sequencing reads in fastq format of metagenomics studies under the BioProject 248 accession numbers PRJNA491626, PRJNA268964 and PRJNA278393 were downloaded from 249 The European Nucleotide Archive (ENA) (https:/www.ebi.ac.uk/ena). Adaptor removal and 250 quality trimming was conducted by Trimmomatic Version 0.39 (USADELLAB.org) ${ }^{26}$. Processed 251 metagenomics reads were mapped to genome assembly of isolated phage using BBTools ${ }^{42}$.

\section{Data availability}

254 The assembled genomes were submitted to the NCBI (https://www.ncbi.nlm.nih.gov/) under the 255 Bioproject accession numbers PRJNA636979 (bacterial genomes) and PRJNA638235 (phage 256 genomes).

\section{$258 \quad$ Results}

\section{Isolation and phenotypic characterization of phages specific for Bacteroides uniformis}


In 8 out of 12 Bacteroides strains belonging to four species the lysis like zones were observed.

261 Subsequently, plaques were successfully propagated from two B. uniformis strains (Data set S1).

262 Circular plaques were formed with diameter ranging from 0.1 to $3 \mathrm{~mm}$ (Figure 1, B). Four 263 seemingly different bacteriophages were isolated (F1-F4). Phages were stable if stored at $4^{\circ} \mathrm{C}$ or -

$26480^{\circ} \mathrm{C}$, at high concentration $\left(10^{11} \mathrm{pfu} / \mathrm{ml}\right)$. Subsequent analysis showed that phages F3 and F4

265 were genetically identical and thus for further experiments only phages F1, F2 and F4 were used.

266 Host range was tested on all Bacteroides strains included in this study (Data set S1). In addition

267 to the initially identified B. uniformis host strains, lysis like forms (Figure 1, C) were observed

268 with additional representatives of B. vulgatus, B. uniformis, and B. ovatus, although we were not

269 able to further propagate the phages.

270 Attempts to isolate potential lysogenic Bacteroides strains from the formed plaques were not

271 successful. Only 10 out of 35 inoculated plates resulted in bacterial growth. These strains were

272 further tested for susceptibility to infection with obtained phages. Experiment was performed

273 three times and no lysogens were detected (discussed in detail below).

274 Transmission electron microscopy (TEM) analysis showed morphology typical of the

275 Siphoviridae family of the Caudovirales with icosahedral heads of about $50 \mathrm{~nm}$ in diameter and 276 approximate tail size of $150 \times 8 \mathrm{~nm}$ (Figure 1, A).

277 Novel B. uniformis phages show high degree of similarity to each other and belong to a new

278 genus

279 The assembled genome lengths of phages F1, F2 and F4 were from 40421 to 40653 bp (Table 1).

$280 \mathrm{G}+\mathrm{C}$ content of phage genome content was $51.8 \mathrm{~mol} \%(\mathrm{~F} 1)$, which is considerably higher than

281 its host genome $\mathrm{G}+\mathrm{C}$ content (46.3 mol \%), obtained from WGS analysis, which is also

282 consistent with Bacteroides uniformis reference stain $\mathrm{G}+\mathrm{C}$ content $^{44}$. 
All four isolated phages were similar one to another (99.83\% similarity) (Table 1). Genomes of phage F1 and F4 differ only in 24 SNP sites, of which 18 are condensed in variable repeat region

F1 but deviates from F1 in an insertion of 19 aa in putative reverse transcriptase gene of the DGR

The isolated phages could not be assigned to any of the known prokaryotic viral clusters using a gene sharing network approach vConTACT2 ${ }^{37}$, implying that so far no similar bacteriophages have been reported (Data set S2 (A) and Figure S1 (B)). Based on no resemblance with phage genera described to date, phages F1, F2 and F4 were classified as a new genus, and for the purpose of this paper provisionally named Bacuni.

TEM based classification of Bacuni phages into Siphoviridae family was additionally confirmed

294 in silico using Virfam server ${ }^{34}$, which identifies proteins of the phage head-neck-tail module and 295 assigns phages to the most closely related cluster of phages within the ACLAME ${ }^{45}$ database 296 (Figure S2).

\section{Genome organization of novel B. uniformis phages}

298 Using automated annotation, 51 open reading frames (ORFs) were predicted in Bacuni genomes.

299 Further functional annotation lead to a prediction of potential functions of 34 genes, which could 300 be divided in five common phage functional groups (Figure 2 and Data set S3).

301 Tandem nucleotide repeats were identified in Bacuni phage ORF for putative phage tail tape measure protein and direct sequencing was conducted to examine whether repeats in phage genome are terminal, which was not the case. Phylogenetic analysis of large terminase subunit 
replication due to clustering into the group of phages with cohesive ends and 3'-single-strand

306 extensions.

307 Nine putative structural proteins were identified, including the major capsid protein, prohead 308 protease, and a large phage tail tape measure protein with observed tandem repeats typical for 309 these proteins ${ }^{46}$ and four transmembrane helices. Large and small subunit of the terminase and 310 portal protein, which together form a packaging function group, were found located in the close 311 proximity of the structural genes. Bacterial cell wall hydrolytic enzyme, a predicted 312 acetylmuramoyl-L-alanine amidase, was identified as a putative lysin.

313 Based on conserved domain search, twelve identified phage genes are putatively involved in 314 DNA metabolism and replication. Additionally, two genes primarily identified as Domains of 315 unknown function (DUF2800 and DUF2815) were recently assigned new putative roles by 316 bioinformatic approach ${ }^{47}$. They are likely to be involved in regulation of phage DNA 317 metabolism. DUF2815 hypothetically functions as single-stranded DNA and DUF2800 as a cis318 regulatory elements or small RNA in phages ${ }^{47}$.

319 Finally, four functionally annotated genes belong to diversity-generating retroelement (DGR).

\section{DGR variability and host tropism}

321 Diversity-generating retroelements are recently described genetic elements that use reverse 322 transcription from a donor template repeat (TR) to a recipient variable repeat (VR) in defined 323 target gene. This generates vast numbers of sequence variants (substitutions) in specific target 324 genes ${ }^{10}$.

325 VR sequences of Bacuni phages are located on genes whose products exhibit DUF1566 and/or 326 Fib_succ_major motifs. The Legionella DGR exemplifies the closest studied DGR ${ }^{19,48}$. DGRs 
327 found in Bacuni phages belong to a group operating on targets exhibiting a C-type lectin fold ${ }^{19}$.

328 This classification and the presence of DGR elements in Bacuni phages were also confirmed with

329 MyDGR, a server for identification and characterization of diversity-generating retroelements ${ }^{35}$.

330 Bacuni phages have two target genes putatively diversified by DGR. First target gene (with

331 detected VR 2) is located on a distant part of the phage F1 genome (6947-7054 bp) while the

332 second target gene with VR $1(20281-20388 \mathrm{bp})$ is found in the immediate neighborhood of the

333 core DGR components including reverse transcriptase (RT) (18141-19544 bp), Avd-accessory

334 protein (19814-20197 bp), and the TR containing gene (19577-19684 bp). The variable repeat

335 gene region, which is diversified, lies at the 3' end of target genes and codes for the last 35 amino

336 acids. Both variable repeats were found at 3' end of the target gene with DUF1566 domain,

337 where also almost all genetic differences between Bacuni phages are located (Table 1, Figure 3).

338 Bacuni phages F1 and F4 differ in 13 amino acids in this region. Target genes in Bacuni phage

339 genomes were found in ORFs that include motifs for cellular adhesion and represent a putative

340 fimbrial tip protein. Identified target genes exhibit high similarity with $60 \%$ or higher coverage

341 (Phyre2) to crystal structure of a fimbrial tip protein (bacova_04982) from Bacteroides ovatus

342 atcc $8483^{49,50}$ that was also identified as a DGR target in metagenomes of human stool samples ${ }^{49}$

344 The observed TR-VR substitutions can be seen in Figure 3 and are, as expected, mutations in 345 adenines. They are most probably the results of induced substitutions mediated by RT (Figure 3).

346 Despite high genetic similarly, isolated Bacuni phages exhibit different host range (Table 1).

347 Since the vast majority of genetic differences was concentrated in VR regions of DGR target 348 genes located in putative fimbrial tip proteins, we propose that DGRs influence host range of 349 Bacuni phages. 


\section{Bacuniphage similarities with other phages and prophages of various anaerobic bacteria}

351 As described above, searches against the NCBI non-redundant database and the Reference Viral

352 Database ${ }^{51}$ showed no similarities of Bacuni phages to any known phages at the nucleotide

353 level. BLASTp search, however, revealed some homology to prophage-related gene products

354 encoded in the genomes within the order Bacteroidales (Table 2; Figure 4).

355 Six putative prophage regions were identified in assembled bacterial genomes with reliable

356 homologies (Table 2; Figure 4).

357 Some of the identified prophage regions were found on contig borders and some assemblies were

358 highly fractioned, thus some parts of prophage genomes could have been left out. The putative

359 functions of retained prophage ORFs were assigned based on conserved protein domains found

360 (Data set S4). The identified putative prophage regions have not been described before.

361 The highest homology (up to $85 \%$ amino acid similarity) to proteins of Bacuni phages was

362 observed in putative prophage regions of B. acidifaciens NM70_E10 and Prevotella sp. P3-122

363 (Figure 4). They share significant protein homology between two thirds of annotated proteins of

364 various functional clusters including the DGR region and its target region overlapping DUF1566

365 domain. However, no homologies were found in its putative lysin and recombinase genes.

366 Protein level homologies found in remaining identified putative prophage regions of Prevotella 367 sp. OH937_COT-195, Porphyromonas gingivicanis COT-022 OH1391, P. cangingivalis JCM

36815983 and Prevotella timonensis UMB0818 were mostly present in structural and packaging

369 functional gene groups (Figure 4).

370 The prevalence of predicted prophage regions identified in initial screening (Table 2) was further 371 examined in Genebank nr-database. Minor nucleotide level similarities of the predicted prophage 
372 regions were found, with a few exceptions. Nucleotide homology (92\%) on 30\% of putative $B$.

373 acidifaciens NM70_E10 prophage region length was found in genomes of $B$. ovatus 3725 D1

374 (CP041395.1), Bacteroides xylanisolvens strain H207 (CP041230.1), and in unidentified phage

375 clone $1013($ JQ680349.1).

376 Whole sequence of predicted $P$. cangingivalis JCM 15983 prophage was also found in the

377 genome of $P$. cangingivalis ATCC 700135 isolated in Finland and in $P$. cangingivalis

378 NCTC12856 collected in 1986 and isolated from fecal sample of Homo sapiens.

Identification of Bacuni phages in Human gut virome database and in associated

381 Genome of Bacuni phage F1 was blasted (blastn) against Human gut virome database (GVD), a 382 novel database composed of 13,203 unique viral populations obtained from gut metagenomes of 383572 individuals from different geographical locations ${ }^{52}$. Matches (roughly $80 \%$ nucleotide 384 similarity over more than $80 \%$ of the Bacuni phages) were found in contigs originating from two studies ${ }^{53,54}$. Data was further tracked to authentic metagenomics data sets that include 386 metagenomes from Western urban societies and traditional communities ${ }^{53,54}$. Search for reads mapping to Bacuni phage genome revealed that Bacuni phages were underrepresented in Western 388 data sets analyzed, but present in data sets of fecal viromes of Cameroonians with gastroenteritis 389 (Data set S5). Up to 6066 reads from metavirome of a Cameroonian ${ }^{54}$ were found to align to 390 Bacuni phage, majority originating from the Kumba region (Data set S5). Further analysis 391 showed that those reads cover 31 of the $40 \mathrm{~kb}$ Bacuni phage F1 genome. 
Assay for detection of lysogenic bacteriophage in B. uniformis host strains was conducted (Data set S1, Figure S4). Three attempts to isolate potential lysogenic host strains from the formed plaques resulted each in roughly 10 viable derivatives of B. uniformis MB18-80 and B. uniformis

397 MB18-33. These derivative strains were retested with all three Bacuni phages. Spot assay showed mixed results: some derivatives were indeed not lysed by any of the phages (representing possible lysogens), while some were resistant to challenging phages but lysed by phages that initially did not lyse the original strain. Thus, this was not a simple lysogenization.

Two derivatives of $B$. uniformis MB18-80, host of phage F4, were further selected for WGS:

402 MB18-80-K, a potential lysogen, that was resistant to infection with all tested phages, and second 403 derivative MB18-80-PH that became susceptible to infection with phages F1 and F2, but resistant 404 to F4 (Data set S1, Figure S4).

405 Genome analysis of B. uniformis MB18-80-K and B. uniformis MB18-80-PH disproved 406 assumptions of lysogenic lifestyle since no parts of Bacuni phage genome were detected in 407 genome of sequenced derivative strains. These results were in agreement with the predicted lytic 408 life style of isolated phages with Phage classification tool set (PHACTS) ${ }^{36}$.

409 Comparison of the obtained B. uniformis derivative genomes to original host strain indicated 410 SNPs in several biologically relevant genes (Table 3, Data set S6). Genome of immune derivative 411 B. uniformis MB18-80-K exhibits SNPs in genes coding for putative restriction enzymes 412 involved in defense mechanism against invading viruses and in outer membrane transporter 413 complexes most likely involved in import of large degradation products of proteins or 414 carbohydrates (Table 3, Data set S6). B. uniformis MB18-80-PH, in which phage tropism 415 switching was observed, exhibited SNPs in partially overlapping set of genes coding for 
416 restriction enzymes, putative porins, peptidoglycan binding proteins, and putative peptidoglycan

417 hydrolase (Table 3, Data set S6).

419 Discussion

420 Bacteroides is one of the most prominent bacterial genera of the human gut microbiome and is

421 known as dietary fiber fermenter that produces short chain fatty acids important for host health

42255,56 . As such it is commonly found in globally conserved core gut microbiota ${ }^{57-60}$.

423 In this study, we describe isolation and characterization of human gut associated phages infecting

424 B. uniformis. As they were essentially not similar to any of the hitherto described phages based

425 on their encoded proteins, we were not able to classify them using VconTACT2. Thus, they may

426 be the first isolated representatives of a new phage genus, provisionally named here a "Bacuni

427 phage".

428 Three isolated phages infected distinct B. uniformis strains. The tropism of Bacuni phages

429 appears to be dependent on interplay of DGR mediated sequence variations of phage fimbrial tip

430 proteins and mutations in host genes coding for outer-membrane proteins. Different host range

431 between genetically very similar Bacuni phages can be explained with SNPs sites condensed in

432 variable repeat of DGR region, located in a putative fimbrial tip protein, a gene presumably

433 involved in cell adhesion and possibly acting as a cell receptor in Bacuni phages. The SNPs are at

434 the C-terminus of two target proteins, at variable repeats that each consist of 35 amino acids.

435 Bacuni phages F1 and F4 differ in 13 amino acids at the variable repeat 2 coded fimbrial protein

436 tip end and infect different hosts, while Bacuniphages F1 and F2, that infect the same host, differ

437 in variable repeat 1 far removed from the DGR region. Given that there are only 6 more SNPs 
438 observed between F1 and F4 outside of DGR, one may conclude that variable repeat 2, located in 439 close proximity of reverse transcriptase, presumably plays a decisive role in Bacuni phage 440 tropism in our experimental setting. These findings correlate with study where metagenomics 441 data set from Human microbiome project (HMP) was screened for DGRs ${ }^{50}$. There, the identified 442 variable regions were also localized in a DUF1566 domain coding genes and the target protein 443 showed high protein homology to a pilin tip from Bacteroides ovatus ${ }^{11,49}$.

444 It appears that DGR contributes to increased adaptability of temperate and lytic phages in such 445 complex communities as the human gut, where multiple species of the same genus and several 446 strains of the same species may coexist. This evolutionary advantage may (indirectly) affect 447 microbial diversity and influence health of the associated mammalian host.

448 To the best of our knowledge, Bacuni phages represent the first DGR-containing lytic phages ${ }^{11}$. 449 Based on protein homologies to here described six putative prophages (Figure 4, Table 2) and 450 their paucity in virome studies, it is plausible that Bacuni phages originate from temperate 451 phages.

452 Viral databases do not contain many genomes of phages infecting dominant gut bacteria and we 453 were initially not able to locate a metagenome/virome that contained sequence reads mapping to 454 Bacuni phages. However, recently published GVD database improves viral detection rates over 455 NCBI viral RefSeq by nearly 60-fold" 52 . Almost complete Bacuni phage genome was found in 456 GVD originating from intestinal viromes of Cameroonians ${ }^{54}$. Weak signal of reads in 457 metagenomes of traditional Peruvian communities and urban Italian gut metagenomes ${ }^{53}$ may 458 indicate that these phages are present at various geographic locations but not abundant enough to 459 be detected with common metagenomics sequencing technologies that are generally not yet 460 optimized to detect bacterial viruses. 
461 Our study sheds light on feasibility of isolation of lytic phages infecting abundant gut bacteria.

462 Lytic phages are suitable for use in phage therapy ${ }^{61-64}$. In vivo studies in mice using commercial

463 phage cocktails showed that phages triggered a cascade reaction that influenced bacterial 464 diversity and composition ${ }^{65}$. Additional further research may provide phages targeting less

465 beneficial bacteria in the intestine with potential therapeutic role on human gut microbiota.

466 In summary, phages described in this study represent a new genus, are the first example of 467 phages using B. uniformis as a host, are one of the rare lytic phages isolated from the gut 468 ecosystem and are the first lytic phages with DGR sequences. Single nucleotide variation in 469 phage DGRs and in the relevant host proteins are described in the context of host specificity 470 pattern changes.

\section{Acknowledgements.}

473 Authors would like to thank Magda Tusek Znidaric for performing TEM.

\section{Disclosure of potential conflicts of interest}

476 Authors report no potential conflicts of interest.

\section{$477 \quad$ Funding details}

478 This work was supported by the Slovenian Research Agency under Grant P3-0387 and Slovenian 479 Research Agency Young Investigators Grant (SH). 


\section{References}

482 1. Sausset R, Petit MA, Gaboriau-Routhiau V, De Paepe M. New insights into intestinal phages.

2. Sordi L De, Lourenço M, Debarbieux L. "I will survive ": A tale of bacteriophage-bacteria coevolution in the gut. Gut Microbes. 2019;10(1):92-99. doi:10.1080/19490976.2018.1474322

3. Aggarwala V, Liang G, Bushman FD. Viral communities of the human gut : metagenomic analysis

4. Minot S, Sinha R, Chen J, et al. The human gut virome : Inter-individual variation and dynamic response to diet The human gut virome : Inter-individual variation and dynamic response to diet. 2011:1616-1625. doi:10.1101/gr.122705.111

5. Minot S, Bryson A. Rapid evolution of the human gut virome. Proc .... 2013;110(30):1245012455. doi:10.1073/pnas.1300833110//

6. Dutilh BE, Cassman N, McNair K, et al. A highly abundant bacteriophage discovered in the unknown sequences of human faecal metagenomes. Nat Commun. 2014;5:1-11. doi:10.1038/ncomms5498

7. Guerin E, Shkoporov A, Stockdale SR, et al. Biology and Taxonomy of crAss-like Bacteriophages, the Most Abundant Virus in the Human Gut. Cell Host Microbe. 2018;24(5):653-664.e6. doi:10.1016/j.chom.2018.10.002

8. Shkoporov AN, Khokhlova E V, Fitzgerald CB, et al. ФCrAss001 represents the most abundant bacteriophage family in the human gut and infects Bacteroides intestinalis. Nat Commun. 2018;9(1):4781. doi:10.1038/s41467-018-07225-7

9. Andrew J. Hryckowian, Bryan D. Merrill, Nathan T. Porter W Van, Treuren, Eric J. Nelson, Rebecca A. Garlena, Daniel A. Russell EC, Martens JLS. Bacteroides thetaiotaomicron-infecting bacteriophage isolates inform sequence- based host range predictions. preprint. 2020. doi:10.32388/nqwmca

10. Cornuault JK, Petit M, Mariadassou M, et al. Phages infecting Faecalibacterium prausnitzii belong to novel viral genera that help to decipher intestinal viromes. 2018:1-14.

11. Benler S, Cobián-Güemes AG, McNair K, et al. A diversity-generating retroelement encoded by a globally ubiquitous Bacteroides phage 06 Biological Sciences 0605 Microbiology. Microbiome. 2018;6(1):1-10. doi:10.1186/s40168-018-0573-6

12. Devoto AE, Santini JM, Olm MR, et al. Megaphages infect Prevotella and variants are widespread in gut microbiomes. Nat Microbiol. 2019;4(4):693-700. doi:10.1038/s41564-018-0338-9

13. Cornuault JK, Moncaut E, Loux V, et al. The enemy from within: a prophage of \&lt;em\&gt;Roseburia intestinalis\&lt;/em\&gt; systematically turns lytic in the mouse gut, driving bacterial adaptation by CRISPR spacer acquisition. bioRxiv. January 2019:575076. doi:10.1101/575076

14. Hawkins SA, Layton AC, Ripp S, Williams D, Sayler GS. Genome sequence of the Bacteroides fragilis phage ATCC 51477-B1. Virol J. 2008;5(Figure 1):1-5. doi:10.1186/1743-422X-5-97

15. Ogilvie LA, Caplin J, Dedi C, et al. Comparative (meta)genomic analysis and ecological profiling of human gut-specific bacteriophage $\varphi \mathrm{B} 124-14$. PLoS One. 2012;7(4):1-17. doi:10.1371/journal.pone.0035053 
16. Gilbert RA, Kelly WJ, Altermann E, et al. Toward understanding phage: Host interactions in the rumen; complete genome sequences of lytic phages infecting rumen bacteria. Front Microbiol. 2017;8(DEC):1-17. doi:10.3389/fmicb.2017.02340

17. Pacífico C, Hilbert M, Sofka D, et al. Natural occurrence of Escherichia coli-infecting bacteriophages in clinical samples. Front Microbiol. 2019;10(OCT):1-18. doi:10.3389/fmicb.2019.02484

18. Sears CL. A dynamic partnership: Celebrating our gut flora. Anaerobe. 2005;11(5):247-251. doi:10.1016/j.anaerobe.2005.05.001

19. Wu L, Gingery M, Abebe M, et al. Diversity-generating retroelements: Natural variation, classification and evolution inferred from a large-scale genomic survey. Nucleic Acids Res. 2018;46(1):11-24. doi:10.1093/nar/gkx1150

20. Liu M, Deora R, Doulatov SR, et al. Reverse transcriptase-mediated tropism switching in Bordetella bacteriophage. Science (80- ). 2002;295(5562):2091-2094. doi:10.1126/science.1067467

21. Doulatov S, Hodes A, Dal L, et al. Tropism switching in Bordetella bacteriophage defines a family of diversity-generating retroelements. Nature. 2004;431(7007):476-481. doi:10.1038/nature02833

22. Krishnamurthy SR, Wang D. Origins and challenges of viral dark matter. Virus Res. 2017;239:136142. doi:10.1016/j.virusres.2017.02.002

23. Bianciotto V, Bondi C, Minerdi D, Sironi M, Tichy H V., Bonfante P. An obligately endosymbiotic mycorrhizal fungus itself harbors obligately intracellular bacteria. Chemtracts. 1998;11(3):206-211. doi:10.1128/aem.62.8.3005-3010.1996

24. Cole JR, Wang Q, Fish JA, et al. Ribosomal Database Project: Data and tools for high throughput rRNA analysis. Nucleic Acids Res. 2014;42(D1):633-642. doi:10.1093/nar/gkt1244

25. Wingett SW, Andrews S. Fastq screen: A tool for multi-genome mapping and quality control [version 1; referees: 3 approved, 1 approved with reservations]. F1000Research. 2018;7(0):1-12. doi:10.12688/f1000research.15931.1

26. Bolger AM, Lohse M, Usadel B. Trimmomatic: A flexible trimmer for Illumina sequence data. Bioinformatics. 2014;30(15):2114-2120. doi:10.1093/bioinformatics/btu 170

27. Magoč T, Salzberg SL. FLASH: Fast length adjustment of short reads to improve genome assemblies. Bioinformatics. 2011;27(21):2957-2963. doi:10.1093/bioinformatics/btr507

28. Bankevich A, Nurk S, Antipov D, et al. SPAdes: A new genome assembly algorithm and its applications to single-cell sequencing. J Comput Biol. 2012;19(5):455-477. doi:10.1089/cmb.2012.0021

29. Gurevich A, Saveliev V, Vyahhi N, Tesler G. QUAST: Quality assessment tool for genome assemblies. Bioinformatics. 2013;29(8):1072-1075. doi:10.1093/bioinformatics/btt086

30. Seemann T. Prokka: rapid prokaryotic genome annotation. Bioinformatics. 2014;30(14):20682069. doi:10.1093/bioinformatics/btu153

31. Finn RD, Bateman A, Clements J, et al. Pfam: The protein families database. Nucleic Acids Res. 2014;42(D1):222-230. doi:10.1093/nar/gkt1223

32. Kelley LA, Mezulis S, Yates CM, Wass MN, Sternberg MJE. The Phyre2 web portal for protein modeling, prediction and analysis. Nat Protoc. 2015;10(6):845-858. doi:10.1038/nprot.2015.053 
33. Almagro Armenteros JJ, Tsirigos KD, Sønderby CK, et al. SignalP 5.0 improves signal peptide predictions using deep neural networks. Nat Biotechnol. 2019;37(4):420-423. doi:10.1038/s41587019-0036-Z

34. Lopes A, Tavares P, Petit MA, Guérois R, Zinn-Justin S. Automated classification of tailed bacteriophages according to their neck organization. BMC Genomics. 2014;15(1):1-17. doi:10.1186/1471-2164-15-1027

35. Sharifi F, Ye Y. MyDGR: A server for identification and characterization of diversity-generating retroelements. Nucleic Acids Res. 2019;47(W1):W289-W294. doi:10.1093/nar/gkz329

36. McNair K, Bailey BA, Edwards RA. PHACTS, a computational approach to classifying the lifestyle of phages. Bioinformatics. 2012;28(5):614-618. doi:10.1093/bioinformatics/bts014

37. Bolduc B, Jang H Bin, Doulcier G, You Z, Roux S, Sullivan MB. vConTACT : an iVirus tool to classify double-stranded DNA viruses that infect Archaea and Bacteria. 2017:1-26. doi: $10.7717 /$ peerj.3243

38. Larkin MA, Blackshields G, Brown NP, et al. Clustal W and Clustal X version 2.0. Bioinformatics. 2007;23(21):2947-2948. doi:10.1093/bioinformatics/btm404

39. Gouy M, Guindon S, Gascuel O. SeaView version 4: A multiplatform graphical user interface for sequence alignment and phylogenetic tree building. Mol Biol Evol. 2010;27(2):221-224. doi:10.1093/molbev/msp259

40. Carver T, Berriman M, Tivey A, et al. Artemis and ACT: viewing, annotating and comparing sequences stored in a relational database. Bioinformatics. 2008;24(23):2672-2676. doi:10.1093/bioinformatics/btn529

41. Sullivan MJ, Petty NK, Beatson SA. Easyfig: a genome comparison visualizer. Bioinformatics. 2011;27(7):1009-1010. doi:10.1093/bioinformatics/btr039

42. Bushnell B, Rood J, Singer E. BBMerge - Accurate paired shotgun read merging via overlap. PLoS One. 2017;12(10):e0185056. https://doi.org/10.1371/journal.pone.0185056.

43. Li H, Handsaker B, Wysoker A, et al. The Sequence Alignment/Map format and SAMtools. Bioinformatics. 2009;25(16):2078-2079. doi:10.1093/bioinformatics/btp352

44. Lorenz Christian Reimer, Anna Vetcininova, Joaquim Sardà Carbasse, Carola Söhngen, Dorothea Gleim, Christian Ebeling, Jörg Overmann, BacDive in 2019: bacterial phenotypic data for Highthroughput biodiversity analysis, Nucleic Acids Research, Volume 47, Issue D1, 08 January 2019, Pages D631-D636, https://doi.org/10.1093/nar/gky879; Bacteroides uniformis. Definitions. 2020. doi:10.32388/9nem1h

45. Leplae R, Lima-Mendez G, Toussaint A. ACLAME: A CLAssification of mobile genetic elements, update 2010. Nucleic Acids Res. 2009;38(SUPPL.1):57-61. doi:10.1093/nar/gkp938

46. Belcaid M, Bergeron A, Poisson G. The evolution of the tape measure protein: Units, duplications and losses. BMC Bioinformatics. 2011;12(SUPPL. 9):S10. doi:10.1186/1471-2105-12-S9-S10

47. Weinberg Z, Lünse CE, Corbino KA, et al. Detection of 224 candidate structured RNAs by Comparative analysis of specific subsets of intergenic regions. Nucleic Acids Res. 2017;45(18):10811-10823. doi:10.1093/nar/gkx699

48. Arambula D, Wong W, Medhekar BA, et al. Surface display of a massively variable lipoprotein by a legionella diversity-generating retroelement. Proc Natl Acad Sci U S A. 2013;110(20):8212-8217. 
49. Xu Q, Shoji M, Shibata S, et al. A Distinct Type of Pilus from the Human Microbiome. Cell. 2016;165(3):690-703. doi:10.1016/j.cell.2016.03.016

50. Ye Y. Identification of diversity-generating retroelements in human microbiomes. Int J Mol Sci. 2014;15(8):14234-14246. doi:10.3390/ijms150814234

51. Goodacre N, Aljanahi A, Nandakumar S, Mikailov M, Khan AS. A Reference Viral Database (RVDB) To Enhance Bioinformatics Analysis of High-Throughput Sequencing for Novel Virus Detection. mSphere. 2018;3(2):1-18. doi:10.1128/mspheredirect.00069-18

52. Gregory AC, Zablocki O, Howell A, Bolduc B, Sullivan MB. The human gut virome database. bioRxiv. January 2019:655910. doi:10.1101/655910

53. Rampelli S, Turroni S, Schnorr SL, et al. Characterization of the human DNA gut virome across populations with different subsistence strategies and geographical origin. Environ Microbiol. 2017;19(11):4728-4735. doi:10.1111/1462-2920.13938

54. Yinda CK, Vanhulle E, Conceição-Neto N, et al. Gut Virome Analysis of Cameroonians Reveals High Diversity of Enteric Viruses, Including Potential Interspecies Transmitted Viruses. Roossinck MJ, ed. mSphere. 2019;4(1):e00585-18. doi:10.1128/mSphere.00585-18

55. Den Besten G, Van Eunen K, Groen AK, Venema K, Reijngoud DJ, Bakker BM. The role of shortchain fatty acids in the interplay between diet, gut microbiota, and host energy metabolism. $J$ Lipid Res. 2013;54(9):2325-2340. doi:10.1194/jlr.R036012

56. Wu GD, Chen J, Hoffmann C, et al. Linking long-term dietary patterns with gut microbial enterotypes. Science (80- ). 2011;334(6052):105-108. doi:10.1126/science.1208344

57. Arumugam M, Raes J, Pelletier E, et al. Enterotypes of the human gut microbiome. Nature. 2011;473(7346):174-180. doi:10.1038/nature09944

58. Arumugam M, Raes J, Pelletier E, et al. Enterotypes in the landscape of gut microbial community composition. Nature. 2013;3(1):1-12. doi:10.1038/nature09944.Enterotypes

59. Mahnic A, Id MR. Different host factors are associated with patterns in bacterial and fungal gut microbiota in Slovenian healthy cohort. 2018:1-17.

60. Qin J, Li R, Raes J, et al. A human gut microbial gene catalogue established by metagenomic sequencing. Nature. 2010;464(7285):59-65. doi:10.1038/nature08821

61. Cieplak T, Soffer N, Sulakvelidze A, Sandris D. A bacteriophage cocktail targeting Escherichia coli reduces E . coli in simulated gut conditions, while preserving a non-targeted representative commensal normal microbiota. Gut Microbes. 2018;0(0):1-9.

doi:10.1080/19490976.2018.1447291

62. Dalmasso M, Strain R, Neve H, et al. Three new Escherichia coli phages from the human gut show promising potential for phage therapy. PLoS One. 2016;11(6):1-16. doi:10.1371/journal.pone.0156773

63. Krylov V, Shaburova O, Krylov S, Pleteneva E. A genetic approach to the development of new therapeutic phages to fight Pseudomonas aeruginosa in wound infections. Viruses. 2012;5(1):1553. doi:10.3390/v5010015

64. Mills S, Hill C, Coffey A. Movers and shakers : Influence of bacteriophages in shaping the mammalian gut microbiota Movers and shakers Infl uence of bacteriophages in shaping the 
mammalian gut microbiota. 2013;(December 2015). doi:10.4161/gutm.22371

646 65. Tetz G V., Ruggles K V., Zhou H, Heguy A, Tsirigos A, Tetz V. Bacteriophages as potential new mammalian pathogens. Sci Rep. 2017;7(1):1-9. doi:10.1038/s41598-017-07278-6

648 


\section{Tables}

650

Table 1. Comparison of general characteristics of isolated phages belonging to a newly defined genus Bacuni.

\begin{tabular}{|c|c|c|c|}
\hline Phage & F1 & F2 & F3 and F4 \\
\hline Bacterial host & $\begin{array}{c}\text { Bacteroides } \\
\text { uniformis } \\
\text { MB18-33 }\end{array}$ & $\begin{array}{c}\text { Bacteroides uniformis } \\
\text { MB18-33 }\end{array}$ & $\begin{array}{c}\text { Bacteroides } \\
\text { uniformis } \\
\text { MB18-80 }\end{array}$ \\
\hline $\begin{array}{c}\text { No. of predicted ORFs } \\
\begin{array}{c}\text { Assembled genome } \\
\text { length (bp) }\end{array}\end{array}$ & 51 & 51 & 50 \\
\hline $\begin{array}{c}\text { G+C content (\%) } \\
\text { Genetic differences } \\
\text { (compared to F1) }\end{array}$ & Reference & 40653 & 40640 \\
& & $\begin{array}{c}16 \text { SNPs in the DGR } \\
\text { and 19 aa insertion in RT } \\
\text { gene }\end{array}$ & $\begin{array}{c}24 \text { SNPs, 18 in } \\
\text { DGR }\end{array}$ \\
\hline & & $\begin{array}{c}\text { RT identical to RT } \\
\text { F1 }\end{array}$ \\
\hline
\end{tabular}

(RT - reverse transcriptase, VR - variable repeat, aa - amino acids, SNP - single nucleotide polymorphism, ORF - open reading frame; DGR: Diversity-generating retroelements) 
Table 2. Comparison of selected genome characteristics between Bacuni phages and putative partially homologues prophage genomes

\begin{tabular}{|c|c|c|c|c|c|c|}
\hline Host strain & Source & $\begin{array}{l}\text { Collection } \\
\text { date and } \\
\text { location }\end{array}$ & $\begin{array}{l}\text { Region } \\
\text { length } \\
\text { (bp) }\end{array}$ & $\begin{array}{c}\text { No. of } \\
\text { Bacuni } \\
\text { homologou } \\
\text { s proteins / } \\
\text { No. of } \\
\text { ORFs }\end{array}$ & $\begin{array}{c}\text { Coverag } \\
\text { e }(\%)- \\
\text { nt } \\
\text { identity } \\
(\%) \\
*\end{array}$ & $\begin{array}{c}\text { Genome location } \\
\text { and } \\
\text { biosample accession }\end{array}$ \\
\hline $\begin{array}{c}\text { Bacteroides acidifaciens } \\
\text { NM70_E10 }\end{array}$ & $\begin{array}{l}\text { Mus } \\
\text { musculu, } \\
\text { colon and } \\
\text { cecum }\end{array}$ & $\begin{array}{l}2016, \\
\text { Toronto, } \\
\text { Canada }\end{array}$ & 44986 & $28 / 48$ & $\begin{array}{c}45 \% \\
- \\
71.06 \%\end{array}$ & $\begin{array}{c}\text { Node } 8 \\
(64227 \ldots \text { 109212) } \\
\text { SAMN10878312 }\end{array}$ \\
\hline Prevotella sp. P3-122 & $\begin{array}{l}\text { Sus scrofa } \\
\text { domesticu } \\
\quad \text { s, feces }\end{array}$ & $\begin{array}{l}2014, \\
\text { Slovenia: } \\
\text { pig farm } \\
\text { Ihan }\end{array}$ & 34280 & $\begin{array}{l}\text { Contig 46: } \\
21 / 35 \\
\text { Contig } 76: \\
5 / 15\end{array}$ & $\begin{array}{c}46 \% \\
- \\
72.82 \%\end{array}$ & $\begin{array}{c}\text { Contig } 46 \\
(44340 \ldots 78619) \\
\text { Contig } 76 \\
(1 \ldots 12007) \\
\text { SAMN07431220 }\end{array}$ \\
\hline $\begin{array}{c}\text { Prevotella sp. } \\
\text { OH937_COT-195 }\end{array}$ & $\begin{array}{l}\text { Canis } \\
\text { lupus, dog } \\
\text { mouth }\end{array}$ & $\begin{array}{l}2012, \\
\text { Leicestersh } \\
\text { ire, UK }\end{array}$ & 38640 & $17 / 47$ & $\begin{array}{c}28 \% \\
- \\
71.82 \%\end{array}$ & $\begin{array}{c}\text { Scaffold20 } \\
(3163 \ldots 37390) \\
\text { SAMN10478691 }\end{array}$ \\
\hline $\begin{array}{c}\text { Porphyromonas } \\
\text { gingivicanis COT-022 } \\
\text { OH1391 }\end{array}$ & $\begin{array}{l}\text { Canis } \\
\text { lupus, dog } \\
\text { mouth }\end{array}$ & $\begin{array}{l}2012, \\
\text { Leicestersh } \\
\text { ire, UK }\end{array}$ & 35922 & $11 / 39$ & $\begin{array}{c}23 \% \\
- \\
70.63 \%\end{array}$ & $\begin{array}{c}\text { Contig } 6 \\
(16379-\ldots 52300) \\
\text { SAMN03004338 }\end{array}$ \\
\hline $\begin{array}{c}\text { Porphyromonas } \\
\text { cangingivalis JCM } \\
15983\end{array}$ & n.a. & $\begin{array}{l}\text { 2014, The } \\
\text { University } \\
\text { of Tokyo }\end{array}$ & 33481 & $17 / 46$ & $\begin{array}{c}33 \% \\
- \\
67.78 \%\end{array}$ & $\begin{array}{c}\text { Node } 1 \\
(3106 \ldots 36586) \\
\text { SAMD00003336 }\end{array}$ \\
\hline $\begin{array}{c}\text { Prevotella timonensis } \\
\text { UMB0818 }\end{array}$ & $\begin{array}{l}\text { Homo } \\
\text { sapiens, } \\
\text { catheter }\end{array}$ & $\begin{array}{c}2015, \\
\text { USA: } \\
\text { Maywood, } \\
\text { IL }\end{array}$ & 37867 & $16 / 47$ & $\begin{array}{c}4 \% \\
- \\
69.19 \%\end{array}$ & $\begin{array}{c}\text { Node } 1 \\
(56177 \ldots 94272) \\
\text { SAMN07511428 }\end{array}$ \\
\hline
\end{tabular}

*Genome coverage Percent of nucleotide identity (discontiguous megablast) compared to F1 
Table 3. Genetic differences in biologically relevant genes of Bacuni phage F4 host MB18-80 and its derivatives that are immune to infection with Bacuni phages or indicate tropism switching pattern.

\begin{tabular}{|c|c|c|c|}
\hline $\begin{array}{c}\text { Putative function } \\
\text { of B. uniformis MB18-80 protein }\end{array}$ & $\begin{array}{c}\text { SNP in } B . \\
\text { NCBI accession* of } \\
\text { closest BLASTp hit } \\
\text { MB18-80 K } \\
\text { (immune) }\end{array}$ & $\begin{array}{c}\text { SNP in } B . \\
\text { uniformis } \\
\text { MB18-80 PH } \\
\text { (switched } \\
\text { tropism) }\end{array}$ \\
\hline $\begin{array}{c}\text { Type I restriction-modification } \\
\text { system specificity (S) subunit }\end{array}$ & WP_117795664.1, & + & + \\
\hline $\begin{array}{c}\text { TonB-linked outer membrane } \\
\text { protein, SusC receptor }\end{array}$ & WP_080597360.1 & + & + \\
\hline $\begin{array}{c}\text { Outer-membrane protein OmpA, } \\
\text { DUF5082 }\end{array}$ & WP_034528676.1, & & + \\
\hline $\begin{array}{c}\text { Putative porin - exopolysaccharide } \\
\text { biosynthesis protein YbjH }\end{array}$ & WP_034528679.1 & & + \\
\hline
\end{tabular}

*BLASTp coverage range from $96 \%$ to $100 \%$, identity from $99.5 \%$ to $100 \%$ 
654

655

656

657

658

659

660

661

662

663

664

665

666

667

668

669

670

671

672

673

674

675

676

\section{Figure legend:}

Figure 1. Lytic Bacuni phages exhibit Siphoviridae morphology. (a) Photograph of Bacuni virion obtained by transmission electronic microcopy (scale bar is $100 \mathrm{~nm}$ ). (b) Plaque morphology of Bacuni phage F1 formed on B. uniformis MB18-33 host lawn after 24 hours incubation in sABB agar overlay. (c) Lysis like zones formed on sABB agar overlay after 24 incubation with host strain Bacteroides vulgatus MB18-32 in double layer agar overlay (spot assay with enrichment sample).

Figure 2. Linear genome map of Bacuni phage F1. Colors of open reading frames correspond to the general predicted functions (see color legend for details). Genes with no functional annotations (hypothetical proteins) are not labeled. Locations of template sequence (TR) and variable repeats of diversity-generating retroelement (DGR) are marked with orange and red rectangles above associated proteins.

Figure 3. Alignment of the TRs and VRs from isolated Bacuni phages. Each nucleotide base is color-coded for visualization of mismatches in the variable repeat. VR2, located in the close proximity of reverse transcriptase represent the region with highest condensation of SNP sites, which most likely influence Bacuni phage host range.

Figure 4. Comparison of genome organization and genomic synteny of Bacuni phages to putative prophage genomes in various bacterial hosts from Bacteroidales. BLASTp sequence homology (40\% similarity and higer) between Bacuni phage F1 and related prophage regions identified in genomes of B. acidifaciens, Prevotella sp., $P$. gingivicanis and P. cangingivalis (see Table 2 for more information) is indicated with a color link. Colors of putative proteins correspond with the general predicted functions (see color legend). 
bioRxiv preprint doi: https://doi.org/10.1101/2020.10.09.334284; this version posted October 10, 2020. The copyright holder for this preprint (which was not certified by peer review) is the author/funder. All rights reserved. No reuse allowed without permission. 


\section{Supplemental Material}

679 Data set S1

680 List of Bacteroides strains, isolated from stool sample and associated phage screening and host 681 range experiments.

682 Data set S2 (A) and Figure S1 (B)

683 Taxonomic anaylsis conduced with vConTACT2 ${ }^{37}$ shows that isolated phages could not be 684 assigned to any of the known prokaryotic viral clusters. Supplemental file S2 contains the 685 Cytoscape network file (B) and the data set (A) with viral clusters made by vConTACT2. In the 686 file the phage F1 is named 3P11 and the phage F4 8POS.

\section{Figure $\mathrm{S} 2$}

688 Classification of the Bacuni phage F1 with respect to other related phages in Aclame (Bacuni 689 phage F1 in text box with red border and white background). According to Virfam server 690 generated protein identification of the phage head-neck-tail module, Bacuni F1 clusters into 691 Siphoviridae of the neck type 1, cluster 3 within the phages in the database ACLAME. The 692 conserved genome organization observed among the phages of the ACLAME database was used 693 to define allowed inter-gene distance intervals ${ }^{34}$. Each cluster with associated number represents 694 a different neck type.

695 Data set S3

696 Putative functions of identified ORFs of Bacuni phages and their closest BLAST hits. Functional 697 annotations for each Bacuni F1 predicted ORF. Function were determined by comparisons to the 698 conserved domain database, Pfam, Phyre2, Virfam and MyDGR. For each ORF best BLASTp hit 
699 accession with the corresponding e-vaule, query coverage and percent identity is listed. Predicted

700 signal peptides and transmembrane domains are included.

\section{$701 \quad$ Figure S3}

702 Phylogenetic analysis of terminase large subunit (TerL) generated with phyML using the 703 maximum likelihood approach and GTR nucleotide substitution model. Bacuni phage TerL 704 clusters into the group of phages with cohesive ends and 3 '-single-strand extensions

705 Data set S4

706 The putative functions of ORFs encoded in 6 identified prophages that share homologous 707 proteins with Bacuni phages. Functions were determined by comparisons to the conserved 708 domain database, Pfam, Phyre2, Virfam and MyDGR. For each ORF best BLASTp hit accession 709 with the corresponding e-vaule is listed

710 Data set S5

711 Number of aligned reads mapping to Bacuni phage F1 in human gut derived metagenomics data 712 sets. The Bacuni phage F1 genome was used as a reference to align reads from whole-community 713 metagenomes using BBtools.

\section{$714 \quad$ Figure S4}

715 Schematic methodologic overview of lysogenic assay conducted to explore Bacuni phage host 716 range.

717 Data set S6 
718 Genetic differences in biologically relevant genes of Bacuni phage F4 host MB18-80 and its

719 derivatives that are immune to infection with Bacuni phages or switched host tropism. Genome

720 location of genes with SNPs and their closest blastp hit accession numbers are provided. 


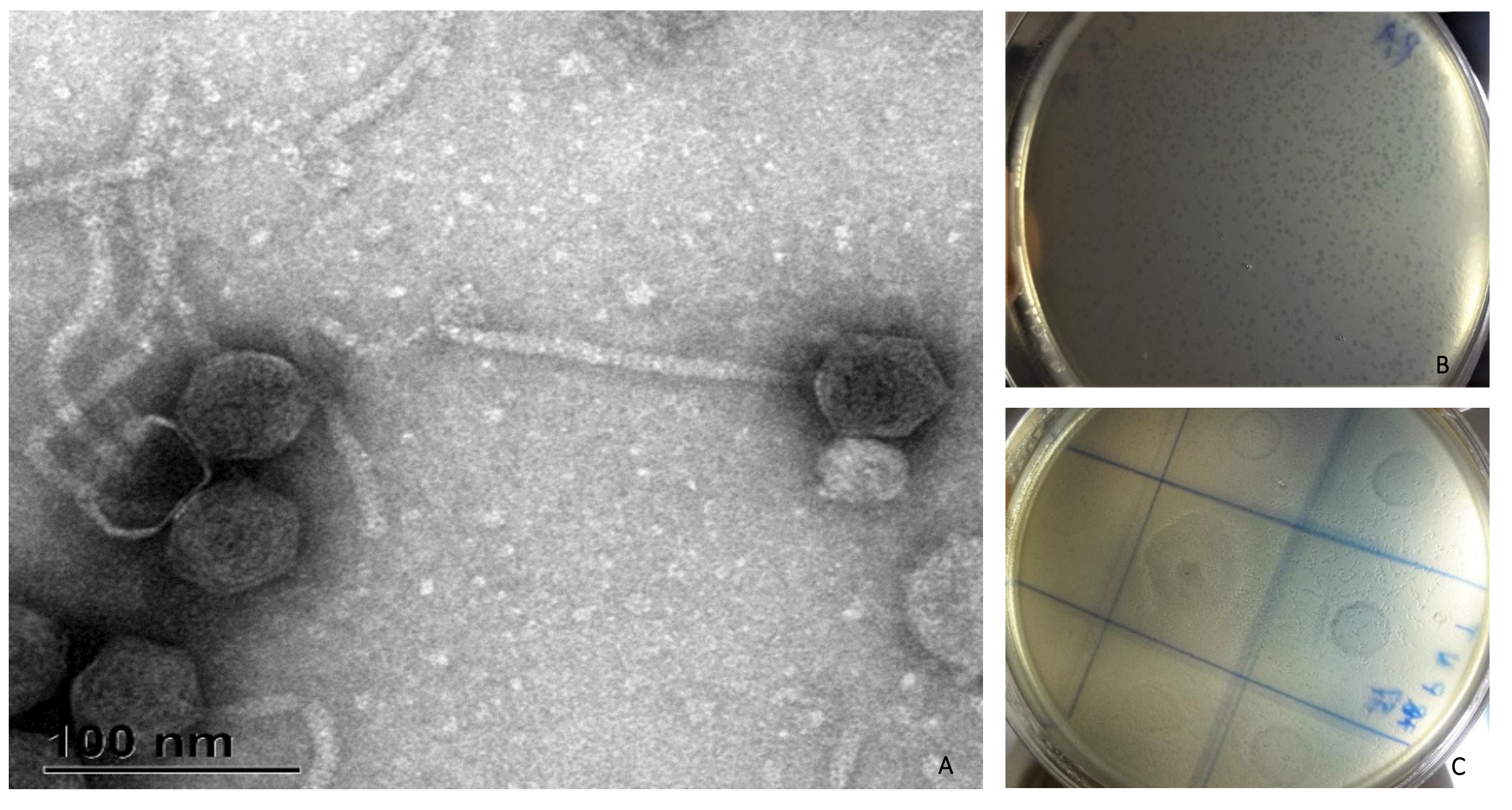




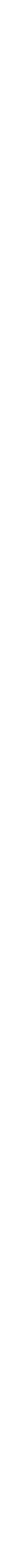



F1_TR

F2_TR

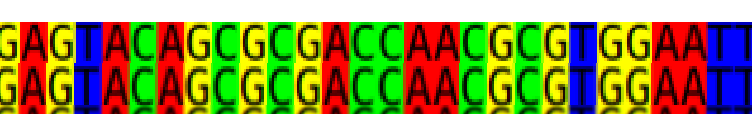

F4_TR

GAACCTCAACAACGGCAACGCGAACAACAACAC GGCGACGAACACGAACAGAG

GAACC CAACAACGGC

VARIABLE REPEATS

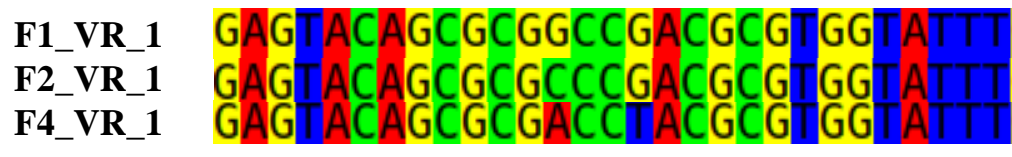

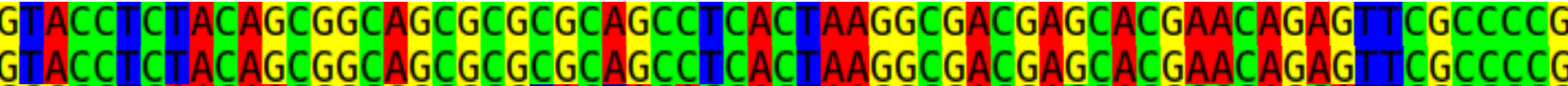

CGCCCCG

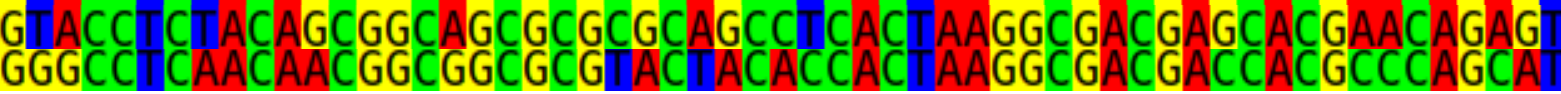

CAGC

F4_VR_1

F1_VR_2

F2 VR_2

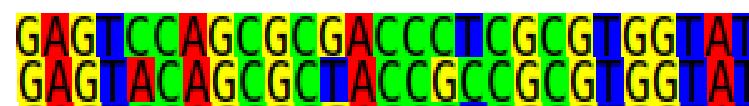

GAACCTCTACAGCGGCTACGCGAACTACGGCGCTGAGGCGACGGGCACGGGCAGAGTCGCCCCG
G ACCTC ACGACGGCGACGCG ACAACGGCAC AAGGCGACGAACACGACCAGAG I CGCCCC

F4_VR_2

GAGICCAGCGCGACCCICGCGIGG

AGGCGACGGGCACGGGCAGAGICGCCCCG 


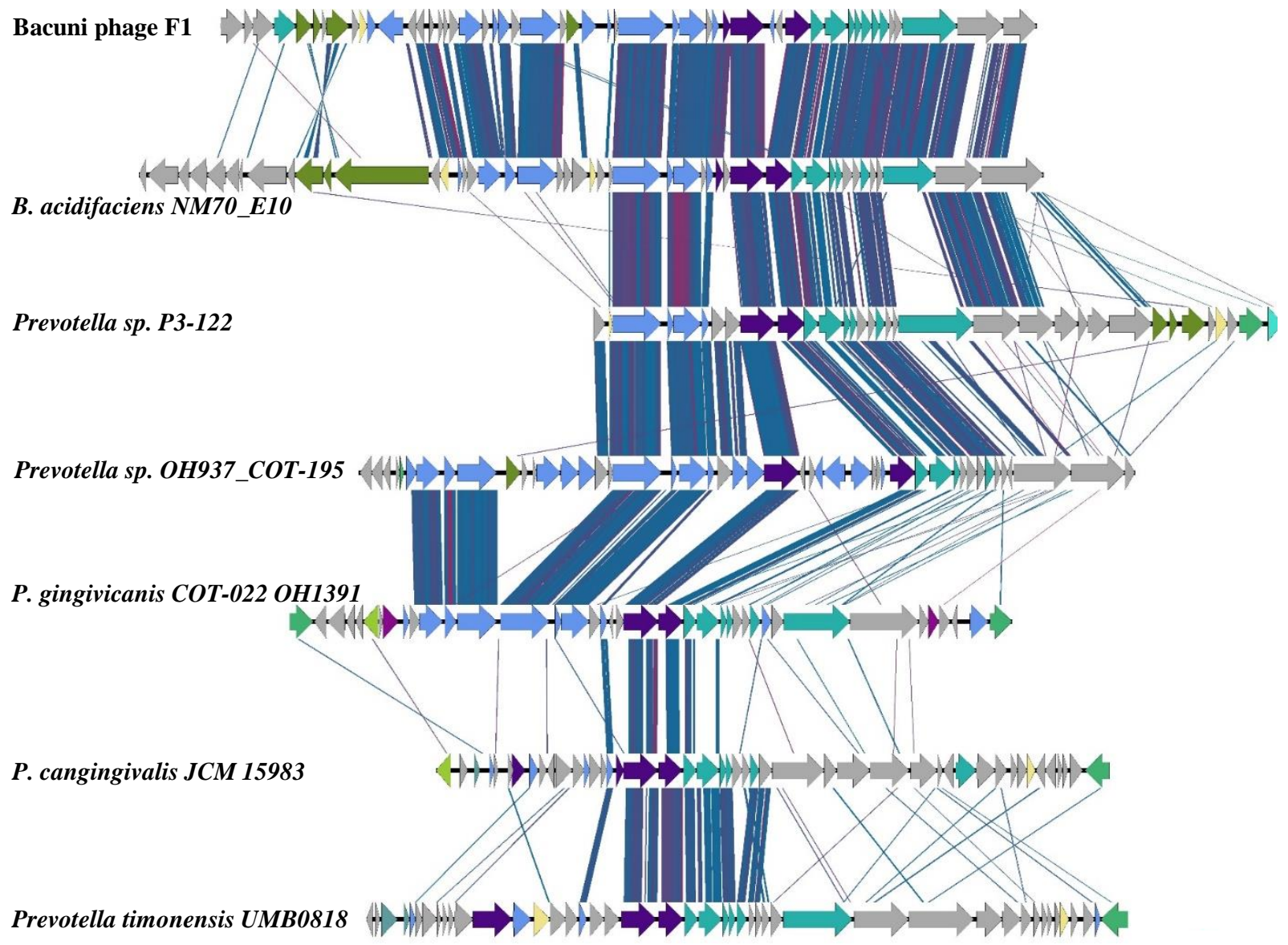

\begin{tabular}{|c|c|c|}
\hline LYSIS & FUNCTION & PHAGE REPRESOR \\
\hline $\begin{array}{l}\text { STRUCTURAL } \\
\text { PROTEINS }\end{array}$ & $\begin{array}{l}\text { UNKNOWN } \\
\text { DGR }\end{array}$ & INTEGRASE \\
\hline $\begin{array}{l}\text { DNA METABOLISM } \\
\text { AND REPLICATION }\end{array}$ & PACKAGING & ANTIREPRESOR \\
\hline
\end{tabular}

Protein sequence homology

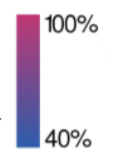

